punched out by the high-speed punch and may be listed off-line if desired. The second and third passes are executed by typing $\mathrm{P} 2$ and $\mathrm{P} 3$, respectively. Output in each case is via the high-speed punch. Between passes, control returns to the library system so assembly may be terminated after any pass.

\section{Symbolic Editor}

The editor may also be run as a background program. Once it has been called it waits with an empty buffer until it receives instructions from the Teletype. The command structure is unchanged except for the addition of a new command, E, which causes exit from the editor to the library system.

\section{Fortran}

The Fortran system has been modified so that high- or low-speed input and output is determined by the form of the statements instead of the settings of the switch register.

The compiler operates as a background program using only high-speed input and output. Error diagnostics must be read off-line from the end of the output tape.
Symbolprint is executed if it is called immediately after compilation.

Calling the operating system merely loads the program into the machine. "GO" must be typed in order to start the program. The program may be repeated any number of times by this command. "PAUSE" statements return control to the library system and "CONT" must be typed in order to continue execution. All Fortran programs must contain a "STOP" statement so that control will eventually return to the library system. If the operating system detects an error at run-time, the diagnostic is printed by the Teletype and control returns to the library system. No recovery is possible.

\section{CONCLUSION}

Two ways of increasing the effectiveness of a small computer in a multiuser research laboraory have been described. Programming has been simplified by the use of modules, and the effective time available on the machine has been increased by the introduction of a simple form of time sharing. The system has been described in terms of a program for controlling a speech perception experiment, but this merely reflects the interests of the authors. The techniques can be applied equally readily to other psychophysical experiments, to the processing of physiological data on-line, and probably to many other situations where the machinery to be controlled operates at human rather than computer speeds.

\section{REFERENCES}

AINSWORTH, W. A. A speech synthesizer controlled by a $P D P-8 / 388$. Proceedings of the Third Decus European Seminar, $1967,1-4$.

AINSWORTH, W. A. First formant transitions and the perception of synthetic semivowels. Journal of the Acoustical Society of America, 1968, 44, 689-694.

AINSWORTH, W. A., \& MILLAR, J. B. Methodology of experiments on the perception of synthesized vowels. Language \& Speech, December 1971, in press.

COOPER, S., DELATTRE, P. C. LIBERMAN, A. M., BORST, J. M., \& GERSTMAN, L. J. Some experiments on the perception of synthetic speech sounds. Journal of the Acoustical Society of America, 1952, 24, 597-606.

HOLMES, J. N., MATTINGLY, I. G., \& SHEARME, J. N. Speech synthesis by rule. Language \& Speech, 1967, 3, 127-143.

\title{
LINC-8 presents and controls visual experiments*
}

\section{GEOFF CUMMING \\ Institute of Experimental Psychology, Oxford, England}

\section{INTRODUCTION \\ The Tachistoscope}

The desire to eliminate the effects of eye movements and to control the presentation time of material used in early span-of-apprehension and visual perception experiments led to the development of the tachistoscope (Woodworth \& Schlosberg, 1954). The most common modern tachistoscopes use electronic timers to switch fluorescent lamps which light one of two or three display fields. Partly silvered mirrors are arranged so that any lighted field appears straight ahead to the S. Material for presentation, such as letters or dot patterns or colored figures, is made up on white cards for mounting in the fields of the tachistoscope. This type of tachistoscope gives excellent definition and contrast of display material, and

* This paper is a later version of a paper presented at the 5th European Seminar of DECUS in Stockholm, September 1969, and published in the Proceedings. allows accuracy and flexibility in the choice of timing parameters. But the use of partly silvered mirrors severely limits the number of fields that may be used, because a sizable proportion of the light reflected from a field is lost every time it is transmitted or reflected by such a mirror, and the use of more fields entails the introduction of more such mirrors into the light paths from the fields to the S's eye. In practice, this means that only two or three arrays may be displayed in a single trial, and frequently one of these arrays must be a masking field, or "visual noise field," to reduce the persistence of afterimages of the preceding array. Also, it is difficult to align different arrays presented on a single trial exactly, as is required in many masking experiments. And it can be very time-consuming to make up the cards for presentation, and then tedious to shuffle them in and out of card holders and change timer settings between trials.

Various devices allowing more than three or four different arrays to be displayed in a single trial have been built. For example, Eriksen and Spencer (1969) used 19 timers, 10 lamps, and 10 optical wave guides to be able to present 10 letters or digits sequentially in one trial. Such devices lose the great flexibility in choice of type, color, size, and position of presentation of the material allowed by the use of cards in mirror tachistoscopes.

The development of television equipment introduced the possibility of displaying in sequence a large number of arrays on a single screen. For example, Averbach and Coriell (1961) used multichannel generating equipment to present three displays in quick succession on a television screen.

\section{Computer-Controlled CRT Displays}

Only with the application of the digital computer to the control of CRT displays was the "many-channel" tachistoscope made possible (Green, 1963). Two important and distinct types of flexibility are offered. First, computer control displays may be built up in space and time by the presentation of any number of elements or whole arrays, each with its own spacing and timing display parameters. Timing is controlled accurately, and different parts of the 
display are aligned exactly. Second, it is much easier to make up and store the material for presentation, and to change display parameters, and so to conduct exploratory experiments.

In addition, there are the general advantages following from the computer control of psychological experiments: Ss generally like to work with a computer, the experiment is better controlled and is more nearly the same for different $\mathrm{Ss}$, responses may be accepted, stored, and analyzed by the computer, and later displays may be changed as the result of preceding responses.

But there are also drawbacks. At present, CRT displays are made up of dot or line patterns and so the legibility of characters is only fair. Brightness cannot be accurately controlled, contrast is not nearly as good as that obtained in a mirror tachistoscope, and only one color may be used. Also, the usable complexity of display may be limited by the speed of the single spot "painting" the display on the scope. In addition, there are the disadvantages of the computer control of psychology experiments: expense, initial programming, and development effort, and the danger that the psychological ends will be subordinated to the computer means.

\section{TSCOPE}

TSCOPE is a set of programs for use with a basic LINC-8, with the usual $4 \mathrm{~K}$ of core store and without an auxiliary clock. It allows accurate timing control of the display of letters, digits, and visual noise fields on the CRT, and flexibility in the selection of parameter values determining timing, order, spacing, and other features of display. Lists of sets of characters may be generated automatically, or typed in, stored on tape, and then displayed in one of two modes. Displays may be presented singly or in groups to form successive experimental trials, each trial being initiated by the S's pressing and releasing a footswitch. Alternatively, a run of trials may be set up so that each trial follows automatically upon the S's keypress response to the previous trial, with either the stimulus-stimulus interval or the response-stimulus interval held constant. At the end of the run, responses and reaction times are stored on tape and later may be typed out or analyzed in various ways.

In describing TSCOPE the following terms will be used:

Character-Any keyboard character accepted by EDIT: the 26 uppercase letters, 10 digits, and space, i, p, - +, $l$, \#. By changing the table of character display codes in CONTROL, it is possible to use any other characters or nonsense figures made up from a 6 by 4 array of dots.

Character set-A sequence of any nine characters: the character set is the basic unit of display material for TSCOPE.

Stimulus list-A sequence of character sets, of any length, possibly interspersed with instruction codes. The stimulus list being prepared or used occupies the first memory bank.

Cycle-The display of a single character set, or of up to eight successive character sets from the stimulus list when $b$ of $\mathrm{CH}$ (see Table 1) is nonzero.

Trial-The display of the entire sequence of one or several character sets and one or many cycles as specified by the current parameter values. The $S$ may trigger each trial by releasing his footswitch, or an experimental run may be formed by the automatic chaining of trials and keypress responses.

se two main TSCOPE programs are introduced here and described in more detail later: (1) CONTROL is the TSCOPE master program, which permanently occupies the second of the three available memory banks (each of which consists of $1 \mathrm{~K}$ of 12-bit words). CONTROL contains the table of current display parameter values and another of character display codes, and builds up a table of responses and reaction times to be stored on tape. It also reads other TSCOPE programs from tape into the third memory bank, in response to console switch settings. (2) DISPLAY usually occupies the third memory bank and is responsible for displaying character sets from the current stimulus list in the first memory bank in accordance with the current parameter values held by CONTROL.

All the other TSCOPE programs are read from tape into the third memory bank when required. The first two allow the formation of stimulus lists: (1) EDIT allows the user to type in any stimulus list which can be used by TSCOPE. Stimulus lists can be made up, stored on tape, read from tape, modified, or typed out for reference. Instruction codes may be inserted between character sets to change display parameters during use of the list, to signal the end of the list, or to call for the next list to be read from tape. (2) GENERATE was developed to supply stimulus lists for visual search and choice reaction time experiments, but has been found to be of more general use as well. The program uses a random-number generator to form stimulus lists of certain restricted types. The user types in eight random digits to initialize the random-number generator, then three alphabets of any size. Then lists of 200 character sets may be generated and stored on tape. The character sets may have from one to nine characters chosen randomly with or without replacement, from the third alphabet. The first two alphabets may provide "target" characters, inserted in random positions, one in each character set.

The next two programs assist in the running of experimental sessions: (1) LOOK provides miscellaneous displaying facilities, the most important of which allows the characters to be used in an experiment, or any special instructions, to be presented on the CRT to the $S$ at the beginning of a session. (2) PAY provides immediate summary information about the S's performance during a run. It uses the LINC floating-point arithmetic package to calculate the mean correct reaction time for a run of trials which has just been completed. It prints this, and the number of errors, and then performs a simple bonus payment calculation using parameters set by the experimenter. Appropriate selection of parameters may be used to encourage strategies of particularly fast or particularly accurate performance.

The final two programs contribute to the analysis of results stored on tape: (1) ANALYZE references the tape block of responses and reaction times gathered during a run, and the stimulus list used for that run. It first types the table of display parameter values used during the run and then types, for each trial, the character set or sets displayed, the S's response, an indication of whether the response was correct or an error, and the reaction time. The typeout thus forms a complete record of the experiment. (2) SETUP also references the four blocks of stimulus list and single block of results corresponding to an experimental run. From these five tape blocks, two tape blocks containing the essential information about the run are formed and stored on tape. The format used allows later analysis by programs written in $4 \mathrm{~K}$-FORTRAN and run by the Linc Executive System (LES, Program L-54, available from the Digital Equipment Computer Users Society).

The two main TSCOPE programs, CONTROL and DISPLAY, will now be described in more detail.

\section{CONTROL}

The experimenter may use a sense switch to have CONTROL display on the CRT a table of parameter labels and their current values. Parameters are then changed by typing the two-letter label and the new four-octal-digit value required; the table of values is changed accordingly. 
Table 1

The list of parameters displayed on the scope by CONTROL. The values are stored, and may be changed by typing in the label and the new value. The definition describes the effect of the parameter on the display of character sets by DISPLAY.

\section{Parameter}

label

Definition of parameter, which has value abcd, four octal digits.

BN

(Block Number)-bcd is the block number of the first block of stimulus list to be read from tape (this is done when $B N$ is first set to abcd); a is number of blocks to be read $(1,2,3$, or 4$)$.

FS

(Fixation Spot)-intensity of the pretrial fixation spot in the center of the scope. The first bit of $a=0$ for constant response-display interval and $=1$ for constant display-display interval.

HI

(Horizontal coordinate, Initial)-horizontal coordinate, 0 to 777 , of the display position of the first character in each set.

VI

(Vertical coordinate, Initial)-vertical coordinate (0 to 777; a multiple of 40 , as required by the hardware character generator) of the display position of the character in each set.

HS

(Horizontal Spacing)-bed is the increment in horizontal coordinate between characters of a set. It is + or - accordingly as a is 0 or 7 .

VS

(Vertical Spacing)-bcd is the increment (a multiple of 40) in vertical coordinate between characters of a set; it is + or - accordingly as a is 0 or 7.

CH

(CHaracter number)-the first bit of $a=1$ if successive character sets are to be displayed on successive cyles; it $=0$ if the same set is to be repeated. This bit has no effect if $\mathbf{C Y}=0$. The second bit of $a=1$ if instruction codes in the stimulus list specifying use of a parameter block are to be obeyed; if it $=0$, they are ignored. The third bit of $a=1$ if each trial is to end with the next character set displayed continuously until turned off, e.g., as an array indicating what type of response is required on this trial; it = 0 otherwise. b ( 0 to 7$)$ is the number of successive character sets to be displayed as a single cycle, and to be repeated as a unit if $\mathrm{CY}$ is nonzero. cd ( 0 to 11 octal) is the number of characters to be displayed from each set. If cd is not 11, spaces are displayed in place of the characters not to be displayed and their corresponding noise fields.

(Character Time)-the delay (0 to 3314 octal msec) between onset of successive characters (or rather, their prenoise fields) within a set.

OR

DR

(ORDeR)-these eight octal digits must together form a permutation of the digits 0 to 7 , giving the temporal order of presentation of the first eight characters of a set (the ninth character is always present last).

(Initial Delay)-the delay (0 to 7776 octal msec) between the $S$ pressing the switch to start the trial and the start of the display (and the interval between cycles if $\mathrm{CY}$ is nonzero).

NA

(Number, Antenoise fields)-the number (0 to 7776) of times the prenoise field (a 6 by 4 chessboard pattern of dots) for each character is scanned. i.e." "painted" onto the scope).

(Delay, Antenoise field)-the delay (0 to 330 octal msec; 0 to about 60 msec for no flicker) between successive scans of the prenoise field; so NA and DA together determine the intensity and duration of the prenoise field.

(Time, Antecharacter)-the delay, 0 to 2000 octal msec, between the offset of the prenoise field and character onset.

NC

(Number, Character)-corresponds to NA, but for the character.

DC

(Delay, Character)-corresponds to DA, but for the character.

TP

(Time, Postcharacter)-the delay, 0 to 2000 octal msec, between character offset and the onset of the postnoise field.

NP

(Number, Postnoise field)-corresponds to NA, but for the postnoise field.

DP

(Delay, Postnoise field)-corresponds to DA, but for the postnoise field.

CY

(CYcle number)-the number, 0 to 7776 octal, of times the basic display (one character set, or more if $\mathrm{b}$ of $\mathrm{CH}$ is nonzero) is cycled.

The current parameter values determine which stimulus list is occupying Memory Bank 1 and being used at the moment, the number, position, and spacing of the characters of any set to be displayed, the temporal order in which the characters are to be displayed, and the delay same set being displayed each time, or with successive sets from the stimulus list being used in sequence.

Table 1 shows the parameters and their effects, and so defines the flexibility of display which TSCOPE allows.

CONTROL occupies Memory Bank 2; this memory bank also provides storage for eight parameter blocks of octal 33 words each. A parameter block consists of nine pairs of vertical and horizontal coordinates, followed by nine time intervals (in milliseconds). The values in any parameter block are set initially and may be changed at any time by an instruction code, followed by a list of the new values, in the stimulus list. Other instruction codes placed immediately before a character set in the stimulus list call for the use of one of the eight parameter blocks when the set is displayed; then the parameters HI, VI, HS, VS, and CT have no effect, as the parameter block does their job. Parameter blocks are useful because they allow variable spacing and variable delays between the characters of a set and because instruction codes may be used to select different parameter blocks, and hence different spacing and delay parameters, for successive character sets.

Trials may be triggered individually-by the S's footswitch. Alternatively, a routine in CONTROL obeys a sense-switch call to set up for the recording of responses and reaction times during a run of trials. Then, once the run has started, successive trials start a fixed interval after the preceding response if Bit 1 of Parameter FS is zero, or after the onset of the preceding trial if this bit is one. When the stop code is encountered in the stimulus list CONTROL writes the responses and reaction times onto tape.

\section{DISPLAY}

Figure 1 provides a skeleton flow chart for DISPLAY, the program occupying Memory Bank 3. DISPLAY obeys any instruction codes encountered in the current stimulus list in Memory Bank 1 and displays the character sets in the stimulus list in accordance with the table of parameter values and one of the parameter blocks (if one is specified) held in CONTROL

Boxes 7 and 8 (see Fig. 1) carry out the actual displaying of character sets and are written to take as little execution time as possible and to take the same time whatever combination of blanks, noise fields, and characters is being displayed. Box 7 takes a little over $1 \mathrm{msec}$ to set up the character display routine (Box 8 ), and this takes 


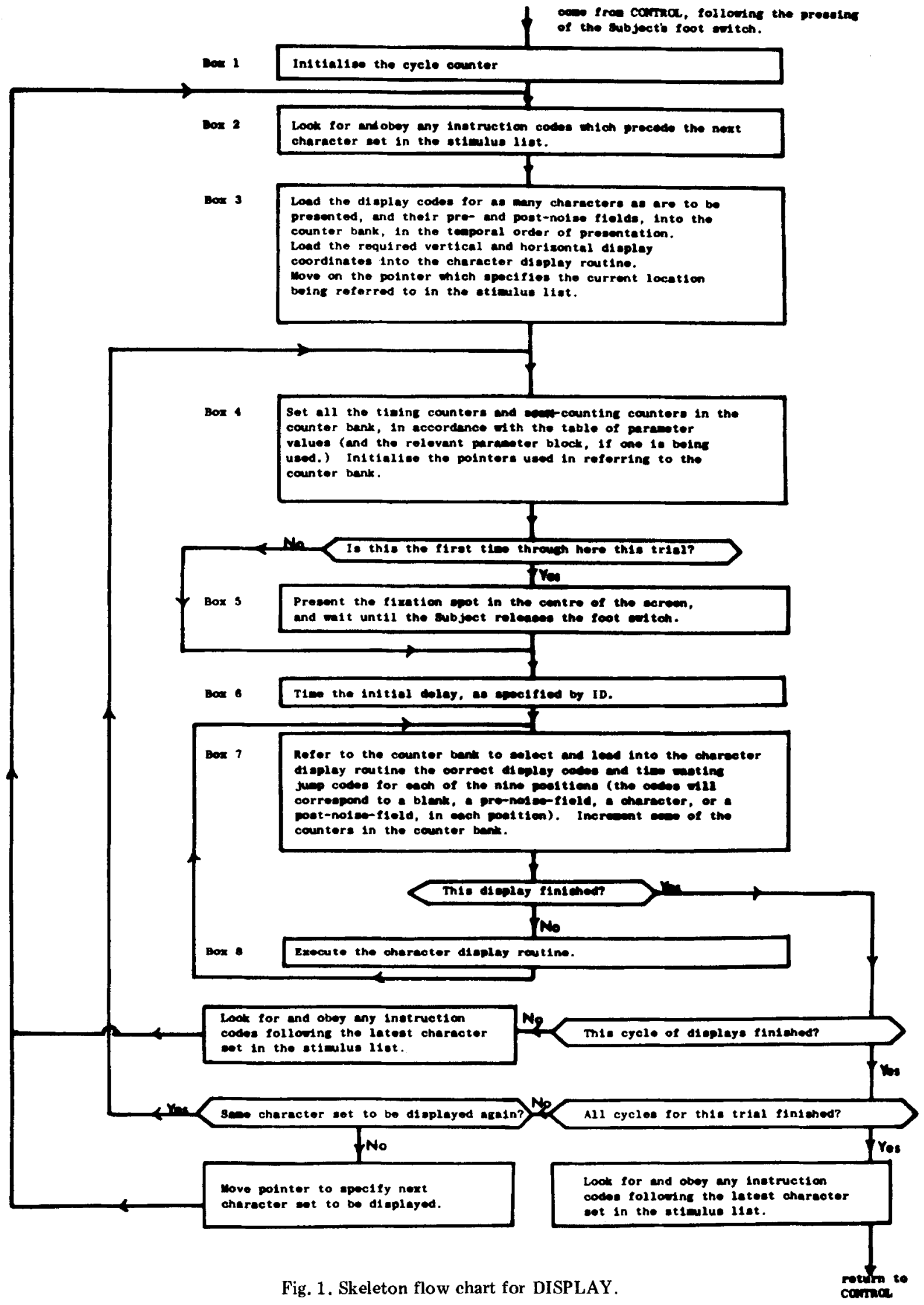

a little over $2 \mathrm{msec}$ in execution, the total execution time for Boxes 7 and 8 being exactly $3.405 \mathrm{msec}$. This display loop time, $3.405 \mathrm{msec}$, is the limit of the timing resolution achieved by TSCOPE, and the timing parameters
(CT, TA, DA, etc.) are divided by 3.405 , rounded to the nearest unit, and then used as counters specifying 
the number of display loops required to make up the specified time interval. Similarly, when reaction times are being measured, the number of display cycles between onset and keypress is counted and stored for later conversion to milliseconds.

TSCOPE relies on software timing because the programs were developed and first used during the 18 months before an auxiliary clock was available. All timing is based on the computer memory cycle time of 1.5 microsec, which is controlled by the interval clock which is based on an RC circuit. The absolute accuracy and the stability of the cycle time has been measured to be much better than $1 \%$, and so is easily accurate enough for the foreseen applications of TSCOPE.

The main difficulty with timing is that the time taken by the hardware character generator to display a character varies from 150 to $260 \mathrm{microsec}$, depending on the number of dots in the 6 by 4 array which must be intensified to form that particular character. Computer display programs refer to tables of display codes to determine which dots to intensity to form a particular character. TSCOPE associates with the display code for each character a delay code which specifies a certain amount of time which must be wasted after every time the character is presented, so that the total display time plus delay time is the same for every character (including "Space").

Returning to the description of DISPLAY, Box 7 consults a section of Memory Bank 3, called the counter bank, to keep track of whether a blank, a prenoise field, a character, or a postnoise field should next be displayed. This must be done independently for each of the nine positions, because when CT is nonzero the display sequence of prenoise field, character, and postnoise field at any position will be out of step with that at each of the other eight positions. Box 7 also counts display cycles as a measure of reaction time and returns control direct to Box 2 for the next trial when the $S$ presses a response key.

Boxes 3 and 4 use parameter values from CONTROL to load the character display routine with nine pairs of position coordinates and to set the initial values of the counters in the counter bank. They use the next set of characters from the stimulus list to select the correct character display codes to load into the counter bank (for use by Box 7 in loading Box 8), and also arrange for the characters to be displayed in the temporal order specified by $O R$ and $D R$.

The tail sections of DISPLAY determine if the present trial has been
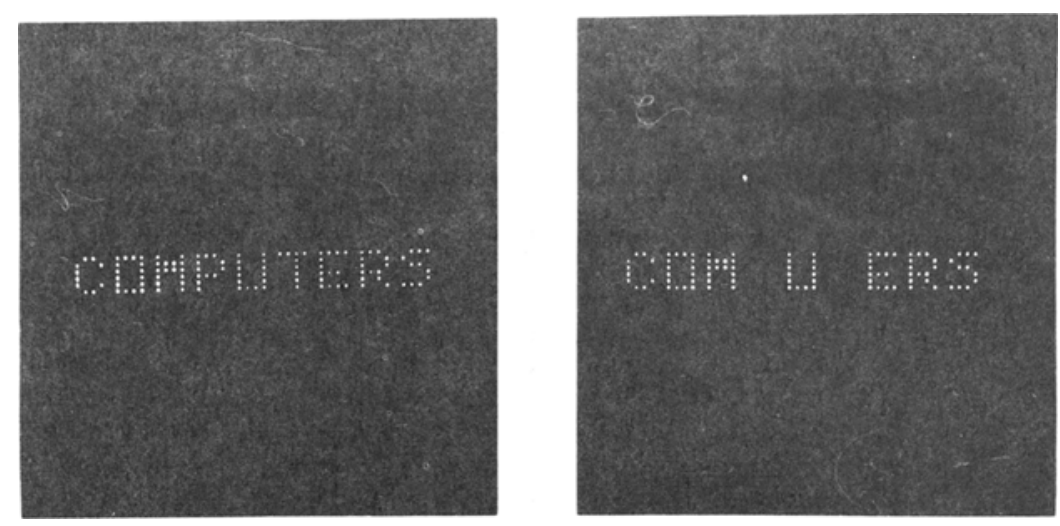

Fig. 2. If the letters of the word COMPUTERS are displayed simultaneously (or rather, within $2 \mathrm{msec}$, the limit imposed by the hardware character generator) then the whole word is seen clearly, as in the left photo. If a 20 -msec delay is introduced between successive letters, and the letters displayed in the temporal order P, T, C, $R, U, M, O, E, S$, then the $P$ and the T are "sequentially blanked" and are not seen by a human observer, although all other letters appear bright and legible. The right photo is a simulation of the effect as reported by observers.

completed, and, if not, initiate a return to Box 4 (to display the same set of characters again) or to Box 2, and eventually make the return to CONTROL at the end of the trial.

\section{TSCOPE IN USE}

The first applications of TSCOPE have been the confirmation of the occurrence of "sequential blanking,"1 and the presentation of 3 by 3 arrays of digits in pilot trials for work in short-term memory. The use of TSCOPE in the single-trial mode, with no response recording, will be illustrated by a short description of each of these applications.

Sequential blanking is a curious phenomenon certain to have theoretical importance and was first described by Mayzner, Tresselt, \& Cohen (1966), who used a CRT display controlled by a PDP-7. (Compared with TSCOPE, the programs written for that installation offer more, but in some ways different, flexibility of display. However, they use a much larger computer installation than does TSCOPE, and control display timing with an auxiliary crystal clock.) To obtain sequential blanking, a single character set, e.g., the word COMPUTERS, is typed into Memory Bank 1 as the stimulus list. All parameters are set to zero, except that $\mathrm{CH}=0011, \mathrm{HI}=0246, \mathrm{VI}=0400$, and $\mathrm{HS}=0024$ (to give the nine positions spaced in a horizontal line in the center of the scope), and $O R=2650$, $\mathrm{DR}=4173$, and $\mathrm{NC}=0011$. This set of values will give a single bright presentation of the word, COMPUTERS, in the center of the scope, lasting for 30 (decimal) msec.

If now $\mathrm{CT}=0024$ is typed in, a delay of 20 (decimal) $\mathrm{msec}$ is introduced between the presentation of successive letters. The spatial order is as before, so that all the letters of the word appear in their correct positions, but $\mathrm{OR}$ and $\mathrm{DR}$ determine the temporal order of presentation. Also as before, each letter is presented for $30 \mathrm{msec}$, but the $P$ is presented first ( $P$, the fourth letter in COMPUTERS, since 0 is the fourth digit in OR, DR). The onset of $P$ is followed successively at 20-msec intervals by the onset of the $\mathrm{T}$ (since 1 is the sixth digit in $O R, D R$ ), the $C$, the $R$, the $U$, the $M$, the $O$, the $E$, and finally the S (see Fig. 2).

The startling finding is that a human $\mathrm{S}$ sees the nine letters as varying markedly in brightness, although each letter is displayed at the same intensity. In fact, with these time parameters and this scrambled presentation order the $\mathbf{S}$ fixating the center of the word fails entirely to see the first two letters presented, the $P$ and the $T$, although these letters are close to his fixation point and he can clearly see other letters in the word.

If ID $=0100$ and $C Y=1000$ are also typed in, the scrambled display of the word is repeated many times, and the effect is found to be compelling and persistent: most letters are seen clearly and brightly, but the $P$ and the $T$ are not seen at all, or at best a very dim unrecognizable flicker is seen in their positions.

Although it is conceivable that sequential blanking could be demonstrated without the use of a computer-controlled display, it seems hardly surprising that its discovery awaited this particular application of the small digital computer. It is clear that the computer makes the rapid sequential presentation of letters (as required for sequential blanking) very 

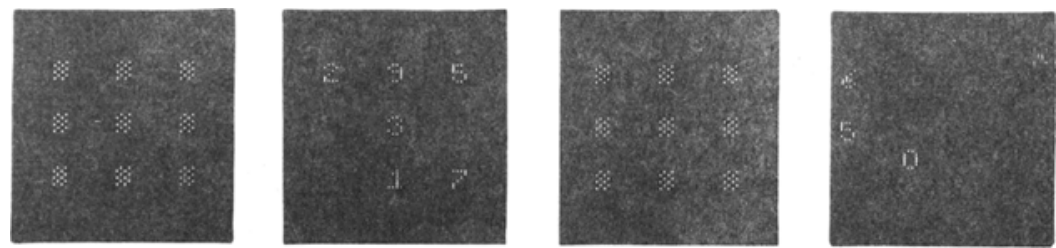

Fig. 3. Arrays used in the short-term memory experiment described in the text. Several hundred such pairs of cards were made up for use with a mirror tachistoscope.

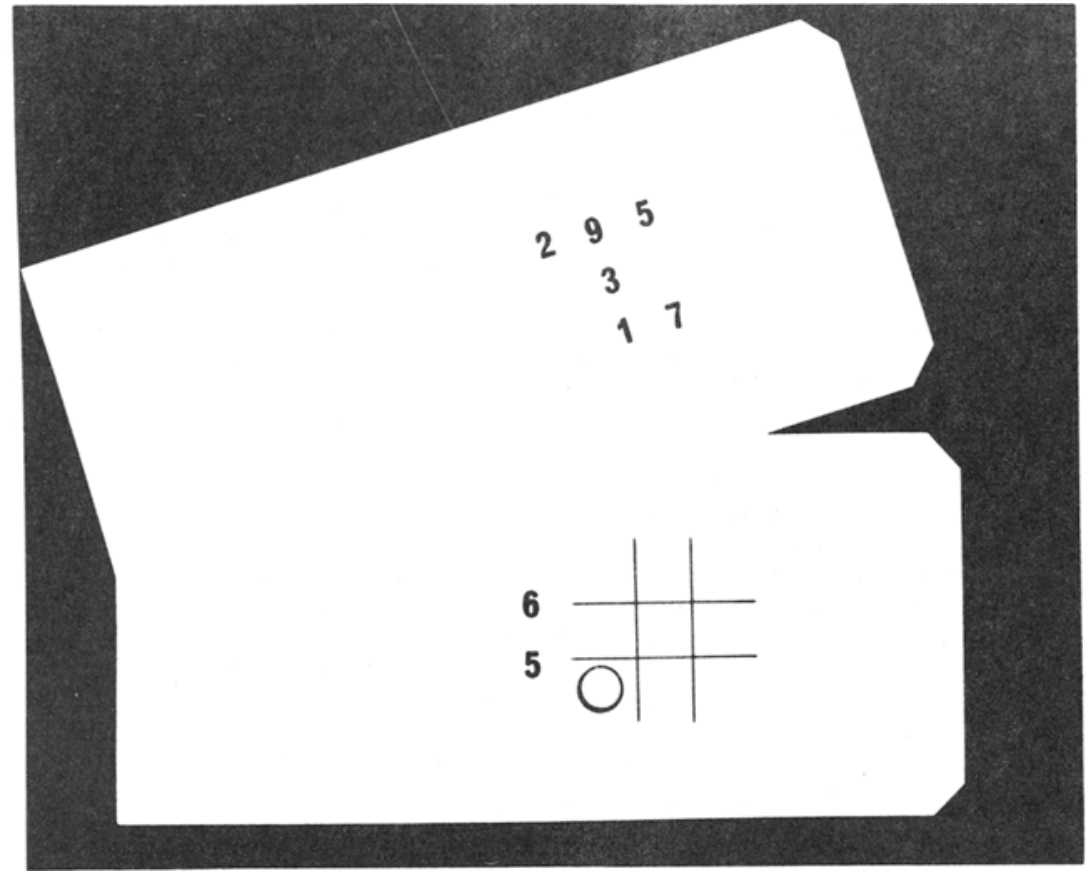

Fig. 4. The same short term memory experiment was run using TSCOPE. The scope displays shown here are, from left to right and in the temporal order of presentation: prenoise field, digit array presented very briefly, postnoise field, and response array presented for as long as required by the $\mathrm{S}$ for response.

easy, but a second type of flexibility made possible by a system such as TSCOPE and the LINC-8 may be almost as important in practice. This is the case with which the material to be displayed and the parameters of display may be changed, by simply typing in new material or new parameter values from the keyboard. It is thus easy to conduct exploratory experiments, varying the stimulus material and display parameters at will.

The other application of TSCOPE to be briefly described has relied heavily on this second type of flexibility. Cumming and Coltheart (1969) used cards and a two-field for the responses required of the $S$ and is displayed continuously (as specified by Bit 3 of $\mathrm{CH}$ ) until the $\mathrm{S}$ presses his footswitch to present the fixation spot preceding the next trial.

TSCOPE not only avoids the card shuffling required to run this experiment on the mirror tachistoscope, but allows easy amendment of the display material. In this case, it was decided to run the experiments using only the digits 1 to 7 ; it took very little time to amend the stimulus list stored on tape compared with the time which would be required to make an entirely new set of tachistoscope cards.

In summary, TSCOPE is suited to the well-controlled display of small amounts of material. Long sequences of presentations can be set up with the $S$ sitting at the CRT initiating each trial with a footswitch, or runs of trials may be presented and the $S$ 's responses and reaction times recorded. TSCOPE should be a useful tool for work in the areas of visual perception of letters and digits which are masked or presented at fast rates, memory span and short-term memory for visually presented characters, and information handling with visual input.

\section{REFERENCES}

AVERBACH, E., \& CORIELL, A. S. Short-term memory in vision. Bell Systems Technical Journal, 1961, 40, 1-3, 309-328.

CUMMING, G., \& COLTHEART, M. Short-term memory: Are item and position information stored independently? Psychonomic Science, $1969,15,216-218$.

ERIKSEN, C. W., \& SPENCER, T. Rate of information processing in visual perception. Journal of Experimental Psychology Monograph, 1969, 79, 2,

GREEN, B. F., JR. Digital computers in research. New York: McGraw-Hill, 1963.

MAYZNER, M. S. The research potential of a computer-based cathode-ray tube display system. Behavioral Research Methods \& Instrumentation. 1968, 1 , 41-43.

MAYZNER, M, A., TRESSELT, M. E., \& COHEN, A. Preliminary findings on some effects of very fast sequential input rates on perception. Psychonomic Science. $1966,6,513-514$.

WOODWORTH, R. S., \& SCHLOSBERG, H, Experimental psychology. London Methuen, 1954.

NOTE

1. Although sequential blanking may be demonstrated on the standard LINC-8 CRT, the effect is better observed on a sereen coated with a fast-decay phosphor. At present a slave CRT with fast-decay phosphor is in use in experiments designed to study the phenomenon. 